\title{
DEVELOPMENT OF TRANSDERMAL DOSAGE FORM USING COPROCESSED EXCIPIENTS OF XANTHAN GUM AND CROSS-LINKED AMYLOSE: IN VITRO AND IN VIVO STUDIES
}

\author{
SILVIA SURINI ${ }^{1 *}$, ASTINA SICILIA ${ }^{1}$, RIKA SOFIANI ${ }^{1}$, SITI KHOIRIAH ${ }^{1}$, UFAIRAH H. INDRIATIN ${ }^{1}$, \\ SANTI PURNA SARI ${ }^{2}$, YAHDIANA HARAHAP ${ }^{3}$
}

${ }^{1}$ Laboratory of Pharmaceutics and Pharmaceutical Technology Development, Faculty of Pharmacy, Universitas Indonesia, Depok, West Java, Indonesia. ${ }^{2}$ Laboratory of Pharmacology, Faculty of Pharmacy, Universitas Indonesia, Depok, West Java, Indonesia. ${ }^{3}$ Laboratory of Bioavailability and Bioequivalence, Faculty of Pharmacy, Universitas Indonesia, Depok, West Java, Indonesia.

Email: silvia@farmasi.ui.ac.id

Received: 02 October 2019, Revised and Accepted: 24 December 2019

ABSTRACT

Objective: A transdermal hydrogel dosage form consists of a three-dimensional polymer network that binds water in large quantities and is used for drug delivery. The study's aim was to prepare coprocessed excipients as a matrix for a transdermal hydrogel containing diclofenac sodium and examine in vitro and in vivo drug penetrations.

Methods: Four types of coprocessed excipients were produced using two methods that combined crosslinking and coprocessing steps. The produced excipients were formulated as transdermal gels containing sodium diclofenac. An in vitro penetration test was then performed using a Franz diffusion cell to pass the drug through a rat skin membrane. An in vivo penetration test was performed by applying the hydrogel to the abdominal skin of male Sprague-Dawley rats and then measuring the plasma drug concentration.

Results: In vitro penetration results showed that the flux from Co-CLA6-XG 1:2, Co-CLA12-XG 1:2, CL6-Co-A-XG 1:2, and CL12-Co-A-XG 1:2 transdermal hydrogels was $655.23 \pm 116.43 \mu \mathrm{g} \cdot \mathrm{cm}^{-2} / \mathrm{h}, 569.08 \pm 26.58 \mu \mathrm{g} \cdot \mathrm{cm}^{-2} / \mathrm{h}, 867.42 \pm 101.27 \mu \mathrm{g} \cdot \mathrm{cm}^{-2} / \mathrm{h}^{-1}$, and $736.99 \pm 15.39 \mu \mathrm{g} \cdot \mathrm{cm}^{-2} / \mathrm{h}^{-1}$. The in vivo study resulted in area under the curve for the Co-CLA6-XG 1:2, Co-CLA12-XG 1:2, CL6-Co-A-XG 1:2, and CL12-Co-A-XG 1:2 transdermal hydrogels was $32.08 \pm 5.40 \mu \mathrm{g} \cdot \mathrm{ml}^{-1} \cdot \mathrm{h}, 34.27 \pm 8.34 \mu \mathrm{g} / \mathrm{ml} \cdot \mathrm{h}, 6.20 \pm 2.90 \mu \mathrm{g} / \mathrm{ml} \cdot \mathrm{h}$, and $14.38 \pm 2.38 \mu \mathrm{g} / \mathrm{mL} \cdot \mathrm{h}$, respectively.

Conclusion: The study results showed that the excipients could be processed to form a matrix within a transdermal hydrogel formulation and deliver sodium diclofenac into systemic circulation in a controlled release manner.

Keywords: Amylose, Xanthan gum, Coprocessed excipient, Transdermal hydrogel, In vitro penetration, In vivo penetration.

(c) 2020 The Authors. Published by Innovare Academic Sciences Pvt Ltd. This is an open access article under the CC BY license (http://creativecommons. org/licenses/by/4. 0/) DOI: http://dx.doi.org/10.22159/ijap.2020.v12s1.FF047

\section{INTRODUCTION}

One aim of research and development of drug delivery systems is to minimize adverse side effects. A common type of pharmaceutical preparation is a transdermal drug delivery system, which delivers a drug through the skin into the systemic circulation. This system has many advantages over oral or injectable drug delivery systems, including avoidance of first-pass metabolism so that a drug can be administered with small therapeutic doses [1]. Moreover, transdermal delivery systems also can reduce undesirable side effects, such as ulceration of the gastrointestinal tract, provide easier administration, and increase patient compliance because of reduced dosing frequency [2]. A type of dosage form that can be used in transdermal drug delivery systems is a hydrogel.

Hydrogels consist of a three-dimensional structure that is able to absorb water or biological fluid in large amounts [1]. Moreover, hydrogels can contain drug molecules in a way that allows the drug to be released slowly [3]. To produce a hydrogel formulation, an excipient is required that is capable of forming a three-dimensional structure that is strong, flexible, and can modulate drug release. Excipients with these functional properties can be obtained through chemical and physical modification of the excipients. One excipient that can prolong drug release is a coprocessed excipient. Coprocessing is a method of mixing an excipient that aims to eliminate unwanted functional properties and maintain or improve the desired functional properties [4]. A coprocessed excipient is obtained by incorporating one of the excipients into the structure of other excipient particles through a physical process, such as codrying.
Among the examples of excipients that can be used for controlled drug release formulations in the form of a hydrogel are high amylose starch and xanthan gum $[5,6]$.

A study conducted by Surini described the development of amylose and xanthan gum excipients as a matrix for sustained-release tablet formulation [7]. The development included modification in crosslinking and coprocessing of the excipients. The resulting excipient was Co-CLA$\mathrm{XG}$, which is a coprocessed excipient of xanthan gum and amylose that was previously cross-linked by sodium trimetaphosphate (STMP). In addition, CL-Co-A-XG was produced, which is a cross-linked excipient of coprocessed amylose and xanthan gum. The study used STMP as a crosslinking agent at concentrations of $6 \%$ and $12 \%$ [7]. In addition, a different variation of amylose and xanthan gum, namely, 1:1, 1:2, and 2:1 were used. As a result, modified cross-linked and coprocessed excipients with ratio (amylose: xanthan gum) of 1:1, 1:2, and 2:1 for each CoCLA6-XG, Co-CLA12-XG, CL6-Co-A-XG, and CL12-Co-A-XG (12 excipient types) were obtained. The Co-CLA-XG excipients were a rough powder (such as fiber) that was voluminous, odorless, and slightly yellowishwhite. The CL-Co-A-XG excipients were fine granules, odorless, white, and slightly yellowish. From those results, the CL-Co-A-XG excipients were characterized by higher viscosity and flow properties than those of Co-CLA-XG. The $6 \%$ concentration of the cross-linked agent gave a greater gel strength of excipient than that of the $12 \%$ concentration. However, both types of excipients were assessed as suitable for controlled release of drugs because such excipients are characterized by high viscosity, high gel strength, and sufficient expansion ability [7]. 
In this study, the excipients of Co-CLA6-XG, Co-CLA12-XG, CL6-Co-A-XG, and CL12-Co-A-XG were used with a 1:2 ratio of amylose and xanthan gum because that ratio is expected to form a solid compact gel. Evaluations of the transdermal hydrogel included physical organoleptic observations, preparation acidity $(\mathrm{pH})$, preparation consistency and thickness, and determination of drug levels in the hydrogel. We also performed in vitro and in vivo penetration tests. The in vitro penetration test was performed using Franz diffusion cells to assess drug penetration through the skin [8]. The in vivo penetration test was performed in male Sprague-Dawley rats as the most representative method to assess drug incorporation into the systemic circulation.

\section{METHODS}

\section{Materials}

Amylose (Shangqiu Kangmedia Bio-Tech, China), xanthan gum (Cargill Bioengineering, Canada), diclofenac sodium (Yung Zip Chemical, Taiwan), STMP (Shangqiu Kangmedia Bio-Tech, China), sodium hydroxide (Merck, Germany), hydrochloride acid (Merck, Germany), sulfuric acid (Merck, Germany), nitric acid (Merck Germany), ascorbic acid (Takeda, Japan), orthophosphate acid (Merck, Germany), potassium dihydrogen phosphate (Merck, Germany), ammonium molybdate tetrahydrate (Merck, Germany), triethylamine high-performance liquid chromatography (HPLC) grade (Merck, Germany), methanol HPLC grade (Merck, Germany), and diltiazem hydrochloride (Piramal Healthcare, India) were used. Other chemicals and solvents were of analytical grade and purchased from commercial suppliers. Sprague-Dawley rats were obtained from Institut Pertanian Bogor (Bogor Agricultural University, Bogor, Indonesia)

\section{Synthesis of Co-CLA6-XG and Co-CLA12-XG (1:2) (method A)}

Synthesis method of cross-linked amylose and coprocessed excipient to give a cross-linked amylose-xanthan gum-based on Cury et al. [9], with modification as stated in Surini et al. [7]. The first stage was synthesis of cross-linked amylose by reacting amylose with STMP as a crosslinking agent. Amylose was dispersed in distilled water while stirring with a magnetic stirrer until perfectly dispersed. Sodium hydroxide $10 \mathrm{~N}$ solution was dripped slowly into the mass to maintain the $\mathrm{pH}$ at 11-12 during the reaction. In another flask, STMP solutions were prepared to produce $6 \% \mathrm{~b} / \mathrm{v}$ for CLA6 and $12 \% \mathrm{~b} / \mathrm{v}$ for CLA 12 . Thereafter, the STMP solution was slowly added to the dispersion of amylose while stirring using a homogenizer (CKL Machinery, Malaysia) at a speed of $3000 \mathrm{rpm}$ for $4 \mathrm{~h}$ and then allowed to stand for $12 \mathrm{~h}$ to ensure completion of the reaction. After that, the suspension of CLA was neutralized by hydrochloride acid $7 \mathrm{~N}$ until a $\mathrm{pH}$ of six was achieved. The suspension washed with ethanol $96 \% \mathrm{v} / \mathrm{v}$ until the filtrate gave a negative result with ammonium molybdate reagent. The residues were dried at room temperature for $48 \mathrm{~h}$. The dried residues were then sieved through a 35-mesh $(500-\mu \mathrm{m})$ sieve.

The second stage was coprocessing for each 3\% CLA (CLA6 and CLA12) with $3 \%$ xanthan gum in distilled water in 1:2 ratio using a homogenizer (CKL Machinery) at $3000 \mathrm{rpm}$ for $30 \mathrm{~min}$. Thereafter, the mass was dried in a drum drier (R. Simon Dryers, UK) at a temperature of $109-112^{\circ} \mathrm{C}$. The produced mass was mashed and sieved by a 35-mesh $(500-\mu \mathrm{m})$ sieve.

\section{Synthesis of CL6-Co-A-XG and CL12-Co-A-XG (1:2) (Method B)}

The first stage was a physical modification by coprocessing amylose with xanthan gum at a concentration of $3 \%$ for each in distilled water in 1:2 ratio. The mass was mixed in a homogenizer (CKL Machinery) at $3000 \mathrm{rpm}$ for $30 \mathrm{~min}$ to obtain a homogeneous mass. The homogeneous mass was dried in a drum drier (R. Simon Dryers) at a temperature of $109-12^{\circ} \mathrm{C}$. The produced mass was mashed and sieved with a $35-$ mesh $(500-\mu \mathrm{m})$ sieve. The second stage was crosslinking of the coprocessed amylose-xanthan gum using the same method as the first stage of method A.

\section{Substitution degree (SD) of the excipients}

The SD was determined by the colorimetry method [10] using two different solutions. The A solution was ascorbic acid 10\%, and the B solution was ammonium molybdate tetrahydrate $0.42 \%$. The sample was placed in a crucible and heated at $600^{\circ} \mathrm{C}$ for $3 \mathrm{~h}$. The ash from the sample was dissolved in sulfuric acid $0.1 \mathrm{~N}$ solution and boiled for $10 \mathrm{~min}$. The solution was then filtered by Whatman 40 paper and diluted with aquadest-sulfuric acid $0.1 \mathrm{~N}$ solutions (1:1). Then, the $\mathrm{A}$ and $\mathrm{B}$ solutions were added to the sample solution. A solution of the sample was mixed and incubated at $45^{\circ} \mathrm{C}$ for $20 \mathrm{~min}$ in a water bath; it was then immediately analyzed using a visible spectrophotometer at a wavelength of $820 \mathrm{~nm}$. The interpretation of the phosphate SD was made by comparison with a calibration curve. The phosphate SD was calculated according to the following equation:

$$
\mathrm{DS}=\frac{162 \mathrm{P}}{3100-102 \mathrm{P}}
$$

where $\mathrm{P}$ is the percentage of phosphate from cross-linked high amylose [11], 162 is the molecular weight of a glucose unit, 3100 is the molecular weight of phosphate, and 102 is the molecular weight of phosphate as $-\mathrm{PO}_{3} \mathrm{Na}$.

\section{Preparation of transdermal hydrogels}

The formulas of Co-CLA6-XG 1:2, Co-CLA12-XG 1:2, CL6-Co-A-XG 1:2, and CL12-Co-A-XG 1:2 transdermal hydrogels are shown in Table 1. The transdermal hydrogels containing diclofenac sodium were prepared by dissolving diclofenac sodium in propylene glycol, then adding it to polyethylene glycol (PEG) 400 and demineralized water. The hydrogel was mixed on a homogenizer at $1000 \mathrm{rpm}$ until it became clear. The drug solution was homogenized in a sonicator for $10 \mathrm{~min}$. Each excipient was dispersed in the drug solution and allowed to stand for $15 \mathrm{~min}$. Subsequently, the mass was mixed in a homogenizer at $1000 \mathrm{rpm}$ and $50^{\circ} \mathrm{C}$ for $15 \mathrm{~min}$ to give a homogenous mass. Then, up to $75 \mathrm{~g}$ of the homogenous mass was placed into a $10 \times 7.5-\mathrm{cm}$ mold and dried in an oven at $50^{\circ} \mathrm{C}$ for $5 \mathrm{~h}$. After cooling, the hydrogel was cut into $1 \times 1-\mathrm{cm}$ sections.

\section{Evaluation of transdermal hydrogels}

Evaluation of the transdermal hydrogels included surface $\mathrm{pH}$, thickness, consistency, and drug content. The surface $\mathrm{pH}$ of the hydrogel was measured by placing the hydrogel in demineralized water for $2 \mathrm{~h}$. The electrode of the $\mathrm{pH}$-meter was then placed on the surface of the hydrogel until it reached equilibrium. The thickness of a transdermal hydrogel section was measured using a caliper. The consistency of the hydrogel was determined using a penetrometer (Herzoo, Germany).

Table 1: Formulation of transdermal hydrogel containing diclofenac sodium

\begin{tabular}{|c|c|c|c|c|c|c|c|c|}
\hline Composition & F1 & F2 & F3 & F4 & F5 & F6 & F7 & F8 \\
\hline $\begin{array}{l}\text { Diclofenac } \\
\text { sodium (g) }\end{array}$ & 5 & 0.5 & 5 & 0.5 & 5 & 0.5 & 5 & 0.5 \\
\hline Co-CLA6-XG (g) & 5 & 5 & - & - & - & - & - & - \\
\hline Co-CLA12-XG (g) & - & - & 5 & 5 & - & - & - & - \\
\hline CL6-Co-A-XG (g) & - & - & - & - & 5 & 5 & - & - \\
\hline CL12-Co-A-XG (g) & - & - & - & - & - & - & 5 & 5 \\
\hline $\begin{array}{l}\text { Polyethylene } \\
\text { glycol } 400 \text { (g) }\end{array}$ & 2.5 & 2.5 & 2.5 & 2.5 & 2.5 & 2.5 & 2.5 & 2.5 \\
\hline $\begin{array}{l}\text { Propylene } \\
\text { glycol (g) }\end{array}$ & 7.5 & 7.5 & 7.5 & 7.5 & 7.5 & 7.5 & 7.5 & 7.5 \\
\hline $\begin{array}{l}\text { Demineralized } \\
\text { water ad (g) }\end{array}$ & 100 & 100 & 100 & 100 & 100 & 100 & 100 & 100 \\
\hline
\end{tabular}

Table 2: Substitution degree of the excipients

\begin{tabular}{ll}
\hline Excipients & Substitution degree \\
\hline CLA6 & $0.08 \pm 0.01$ \\
CLA12 & $0.10 \pm 0.00$ \\
CL6-Co-A-XG & $0.10 \pm 0.00$ \\
CL12-Co-A-XG & $0.17 \pm 0.04$ \\
\hline
\end{tabular}

Each point represents mean \pm standard deviation $(n=3)$ 
Table 3: Properties of the transdermal hydrogels

\begin{tabular}{llllll}
\hline Hydrogel & Formula & Surface $\mathbf{~ H ~}$ & Thickness (mm) & Consistency (yield value) & Drug content in each hydrogel (mg) \\
\hline Co-CLA6-XG & F1 & $7.52 \pm 0.05$ & $8.18 \pm 0.12$ & $5537.71 \pm 102.09$ & $48.15 \pm 0.59$ \\
& F2 & $7.21 \pm 0.04$ & $7.20 \pm 0.38$ & $5679.59 \pm 434.79$ & $5.36 \pm 0.05$ \\
Co-CLA12-XG & F3 & $7.48 \pm 0.30$ & $8.00 \pm 0.27$ & $6150.01 \pm 171.85$ & $48.03 \pm 1.45$ \\
& F4 & $6.96 \pm 0.16$ & $7.15 \pm 0.05$ & $6002.12 \pm 422.85$ & $5.19 \pm 0.13$ \\
CL6-Co-A-XG & F5 & $7.18 \pm 0.12$ & $7.55 \pm 0.20$ & $5752.06 \pm 109.18$ & $50.74 \pm 2.82$ \\
& F6 & $7.35 \pm 0.14$ & $7.40 \pm 0.33$ & $6858.55 \pm 103.08$ & $5.06 \pm 0.06$ \\
CL12-Co-A-XG & F7 & $7.25 \pm 0.02$ & $7.07 \pm 0.09$ & $5578.43 \pm 207.51$ & $50.82 \pm 0.88$ \\
& F8 & $7.33 \pm 0.02$ & $7.27 \pm 0.15$ & $6386.91 \pm 614.70$ & $5.06 \pm 0.06$ \\
\hline
\end{tabular}

Each point represents mean \pm standard deviation $(n=3)$

The diclofenac sodium content in the hydrogel was determined using an ultraviolet (UV) spectrophotometer. Each hydrogel was dissolved in phosphate buffer $\mathrm{pH}$ 7.4. The solution was then homogenized in a sonicator to ensure that the diclofenac sodium was completely dissolved in the phosphate buffer pH 7.4. Hydrogel F1, F3, F5, and F7 were diluted to $1 / 100$ of their initial concentrations with phosphate buffer $\mathrm{pH}$ 7.4, and F2, F4, F6, and F8 were diluted to $1 / 10$ of their initial concentrations with phosphate buffer $\mathrm{pH}$ 7.4. Each sample solution was measured by UV spectrophotometry at a wavelength of $276 \mathrm{~nm}$. Each hydrogel was assayed 3 times.

\section{In vitro penetration study}

The methods used for animal sacrifice for in vitro and in vivo penetration studies were approved by the Ethics Committee of the Faculty of Medicine, Universitas Indonesia (approval no. 224/UN2. FI/ETIK/2015). Sprague-Dawley rats were housed under standard conditions of temperature, relative humidity, and light. Unless otherwise specified, food, and water were given ad libitum.

The in vitro penetration test method was based on that of Klimes et al. [12] with slight modifications. The penetration of diclofenac sodium through the skin was measured using Franz diffusion cells. The receptor compartment was filled with a solution of phosphate buffer $\mathrm{pH} 7.4$, and the temperature was maintained at $37 \pm 0.5^{\circ} \mathrm{C}$. The speed of the magnetic stirrer was set to $250 \mathrm{rpm}$. The abdominal skins were collected from the Sprague-Dawley male rats, which were approximately $8 \mathrm{w}$ old and weighed $\pm 200 \mathrm{~g}$. The skins were placed between the donor and the receptor compartment with the dermal side in contact with the receptor medium. The hydrogels (F1, F3, F5, and F7) were placed in the donor compartment. As much as, $0.5 \mathrm{ml}$ of the sample solutions was withdrawn at each time interval from the receptor and immediately replaced with the same amount of $\mathrm{pH} 7.4$ phosphatebuffered solution. The amount of diclofenac sodium in the sample was then measured using a UV spectrophotometer (Shimadzu, Japan) at a wavelength of $276 \mathrm{~nm}$.

\section{In vivo penetration study}

The method was based on Sintov and Botner [13] with slight modifications. The experiment used 8-w old Sprague-Dawley male rats $(n=6)$ for each hydrogel (F2, F4, F6, and F8). The rats were anesthetized by intra-peritoneum injection of urethane $(1000 \mathrm{mg} / \mathrm{kg})$. The abdominal skins of the rats were shaved, and each hydrogel (F2, F4, F6, and F8) was placed on skin with plaster to keep the hydrogel on the surface of the abdomen during the test. As much as, $0.5 \mathrm{ml}$ of a blood sample was collected from the sinus orbital of the eye at each time interval and then inserted into a microtube that had been loaded with heparin. Blood samples were centrifuged at 10,000 rpm for $20 \mathrm{~min}$ to separate the blood plasma from blood cells.

\section{Analysis of diclofenac sodium in rat plasma}

Diclofenac sodium in rat plasma was analyzed by HPLC (Shimadzu) equipped with a photometric diode array detector at a wavelength of $283 \mathrm{~nm}$. The $250 \times 4.6 \mathrm{~mm} \mathrm{C}_{18}$ reversed-phase column had a particle size of $5 \mu \mathrm{m}$. The mobile phase was a mixture of methanol: bidistilled water containing $1 \%$ triethylamine $\mathrm{pH} 6.5$ by adding orthophosphoric acid $(80: 20)$ at a flow rate of $0.5 \mathrm{ml} / \mathrm{min}$. Before analysis, as much as
Table 4: Flux values and the cumulative amount of the penetrated drug

\begin{tabular}{lllll}
\hline Hydrogel & Formula & $\mathbf{J}\left(\boldsymbol{\mu g} \cdot \mathbf{c m}^{-2} / \mathbf{h}^{-\mathbf{1}}\right)$ & $\mathbf{Q}\left(\boldsymbol{\mu g} . \mathbf{c m}^{-\mathbf{2}}\right)$ & $\mathbf{\%} \mathbf{Q}$ \\
\hline Co-CLA6-XG & F1 & $655.23 \pm 116.43$ & $7.629 \pm 711$ & $23.66 \pm 4.10$ \\
Co-CLA12-XG & F3 & $569.08 \pm 26.58$ & $6.842 \pm 467$ & $19.24 \pm 0.81$ \\
CL6-Co-A-XG & F5 & $867.42 \pm 101.27$ & $10.438 \pm 390$ & $35.41 \pm 9.29$ \\
CL12-Co-A-XG & F7 & $736.99 \pm 15.39$ & $8,994 \pm 105$ & $31.26 \pm 0.33$ \\
\hline
\end{tabular}

Each point represents the mean \pm standard deviation $(n=3)$. J represents the transdermal flux, and $Q$ is the cumulative amount of drug penetrated through the rat skin

$250 \mu \mathrm{l}$ of plasma sample was added with $100 \mu \mathrm{l}$ of internal standard (diltiazem hydrochloride $100 \mathrm{ppm}$ ) and mixed on a vortex mixer for $10 \mathrm{~s}$. Thereafter, the sample was added to $250 \mu \mathrm{l}$ methanol HPLC grade and mixed on a vortex mixer for $2 \mathrm{~min}$ and centrifuged at 10,000 rpm for $10 \mathrm{~min}$ to precipitate the proteins. Furthermore, as much as $20.0 \mu \mathrm{l}$ of supernatant was injected into the HPLC instrument under the selected conditions. The method was partially validated by the European Medicine Agency [14].

\section{RESULTS AND DISCUSSION}

SD of the excipients

The SD was calculated as the degree of substitution of hydroxyl groups by phosphate groups from STMP in the amylose and xanthan gum. Inorganic phosphate was produced by heating the excipient to $600^{\circ} \mathrm{C}$. Inorganic phosphate was reacted with ammonium molybdate tetrahydrate in acid solution to produce a phosphomolybdate complex, which was then reduced by ascorbic acid to produce a blue color [15].

Table 2 shows the differences in the SD between the excipients. The SD values were determined for CLA6, CLA12, CL6-Co-A-XG, and CL12-CoA-XG. An SD of 0.08 indicated substitution of eight phosphate groups for hydroxyl groups in every 100 anhydroglucose units of amylose and/or xanthan gum. An SD of 0.10 indicated a substitution of 10 phosphate groups for hydroxyl groups in every 100 anhydroglucose units of amylose and/or xanthan gum. CL6-Co-A-XG has ten phosphate groups, and CL12-Co-A-XG had an SD of 0.17 indicating 17 substituted hydroxyl groups in every 100 anhydroglucose units of amylose and/ or xanthan gum. From this data, we concluded that increasing the concentration of STMP increased the SD of the excipient. The SD of each excipient did not change its physical characteristics but did affect its functional characteristics, such as the swelling index, gelling strength, and viscosity, which could affect drug release [7].

\section{Evaluation of transdermal hydrogels}

Table 3 shows the evaluation results of the transdermal hydrogels' properties, including surface $\mathrm{pH}$, hydrogel thickness, consistency value, and drug content in each hydrogel. The surface $\mathrm{pH}$ of the transdermal hydrogel was in the range of the limits of skin $\mathrm{pH}$ (5.6-7.5), which indicated that the transdermal hydrogel would not cause local irritation of human skin [16]. The surface $\mathrm{pH}$ of the transdermal hydrogel also was affected by the other components, such as diclofenac sodium, PEG 400, and propylene glycol. As shown in Table 3, there is a slight 
Table 5: Pharmacokinetic parameters of the transdermal hydrogels containing diclofenac sodium

\begin{tabular}{lllllll}
\hline Hydrogel & Formula & $\mathbf{C}_{\max }(\boldsymbol{\mu g} / \mathbf{m l})$ & $\mathbf{T}_{\max }(\mathbf{h})$ & $\mathbf{A U C}_{\mathbf{0}-\mathbf{1 2}}(\boldsymbol{\mu g} / \mathbf{m l} \mathbf{h})$ & $\mathbf{K}_{\mathbf{e}}\left(\mathbf{h}^{-1}\right)$ & $\mathbf{t}_{\mathbf{1} / \mathbf{2}}(\mathbf{h})$ \\
\hline Co-CLA6-XG & F2 & $4.35 \pm 0.94$ & $1.00 \pm 0.00$ & $32.08 \pm 5.40$ & $0.10 \pm 0.03$ & $11.47 \pm 4.04$ \\
Co-CLA12-XG & F4 & $4.87 \pm 1.06$ & $1.00 \pm 0.00$ & $34.27 \pm 8.34$ & $0.12 \pm 0.03$ & $7.96 \pm 1.90$ \\
CL6-Co-A-XG & F6 & $2.87 \pm 0.48$ & $0.50 \pm 0.11$ & $6.20 \pm 1.19$ & $11.89 \pm 2.67$ \\
CL12-Co-A-XG & F8 & $4.34 \pm 0.58$ & $0.50 \pm 0.00$ & $14.38 \pm 2.38$ & $0.18 \pm 0.04$ & $5.29 \pm 1.50$ \\
\hline
\end{tabular}

AUC: Area under the curve, MRT: Mean residence time, $\mathrm{K}$ : Elimination constant. Data are expressed as mean \pm standard error, $\mathrm{n}=6$

difference in thickness among the hydrogels, which is associated with water content in each hydrogel (data not shown). In addition, the produced transdermal hydrogels had a hard consistency according to Sherman [17]. The hardest consistency was observed for CL6-CoA-XG followed by CL12-Co-A-XG, Co-CLA12-XG, and Co-CLA6- XG. The produced transdermal hydrogels showed rigid form but still contained water. The drug content of diclofenac sodium in the transdermal hydrogel was evaluated by UV-visible spectrophotometry at $276 \mathrm{~nm}$. Compared with the drug content in the initial formula, the transdermal hydrogels contained $95-105 \%$ of diclofenac sodium, because the F1, F3, $\mathrm{F} 5$, and F7 should contain $\pm 50 \mathrm{mg}$ for in vitro penetration study and the F2, F4, F6, and F8 should contain $\pm 5 \mathrm{mg}$ for in vivo penetration study.

\section{In vitro penetration study}

Fig. 1 shows the penetration profiles of diclofenac sodium from the transdermal hydrogels across through the rat skin membrane. The flux value and cumulative amount of drug that penetrated through the skin among the four hydrogels, comprising different polymers, are shown in Table 4 . The diffusion of diclofenac sodium through the skin was passive with the concentration gradient as the driving force. The diffusion was affected by many factors, such as the solubility of diclofenac sodium and the thickness of the stratum corneum [18]. Diclofenac sodium in water solution can be hydrolyzed to diclofenac acid, which will increase the partition coefficient of the active substance so that the penetration of the drug can also be [14]. The mechanism of drug permeation through the skin begins by swelling of the hydrogel. The receptor compartment, which consists of phosphate buffer $\mathrm{pH}$ 7.4, caused hydration of the rat skin. The transdermal hydrogel, which consisted of a polymer matrix, could be wetted by absorbing fluid as it contacts the skin. This process causes the hydrogel to swell so that diclofenac sodium diffuses through the three-dimensional network of the polymer. During that process, the gradient concentration between the rat skin and hydrogel is high, which causes diclofenac sodium to permeate through the skin.

The highest cumulative amount of drug penetrated through the skin was achieved by the CL6-Co-A-XG 1:2 hydrogel (F5). The crosslinking in CL6-Co-A-XG (1:2) may occur between amylose and xanthan gum. The more crosslinking indicates high crosslinking density, which means the network was closely woven. In this case, the chain sections of the crosslinks were short and anchored by many points. Therefore, the polymer became less flexible and more rigid [19]. This characteristic makes diffusion of the drug easier through the matrix.

The Co-CLA6-XG 1:2 (F1) and Co-CLA12-XG 1:2 (F3) hydrogels gave levels of penetration of diclofenac sodium $<30 \%$ because the three-dimensional networks of hydrogels F1 and F3 consisted of some closed-hydrogen bonds and high gel strength that slowed drug release [7]. Dumoulin et al. stated that drug release could be controlled by crosslinking amylose, the degree of which was limited in CLA-3 to CLA-11, and the maximum drug release time was observed in CLA- 6 (as long as 20-24 h) [20]. Furthermore, Lanaerts et al. [20] stated that the increase in the degree of crosslinking from CLA- 6 to CLA-20 correlated with the decrease in crystal properties, which can lead to a decreasing ability to form a double-helix structure and acceleration of matrix hydration [20].

The percentage of cumulative drug penetrations of all hydrogels through the skin was $<50 \%$, which was caused not only by the ability of the polymer to slow drug release but also the characteristics of the

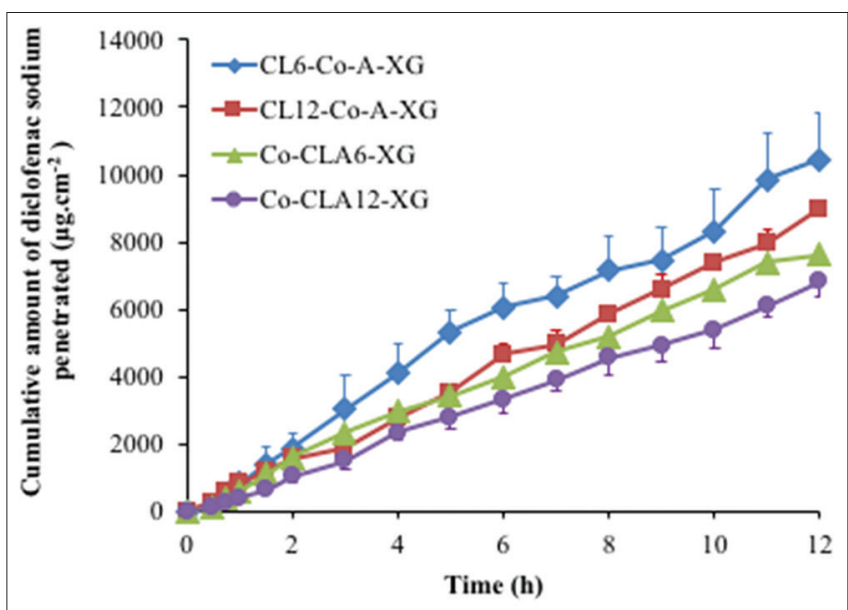

Fig. 1: In vitro percutaneous penetration profiles of diclofenac sodium through excised rat skins after application of transdermal hydrogel prepared from different excipients. Green, purple, blue, and red lines represent F1, F3, F5, and F7, respectively. Each point represents the mean \pm standard deviation; $n=3$

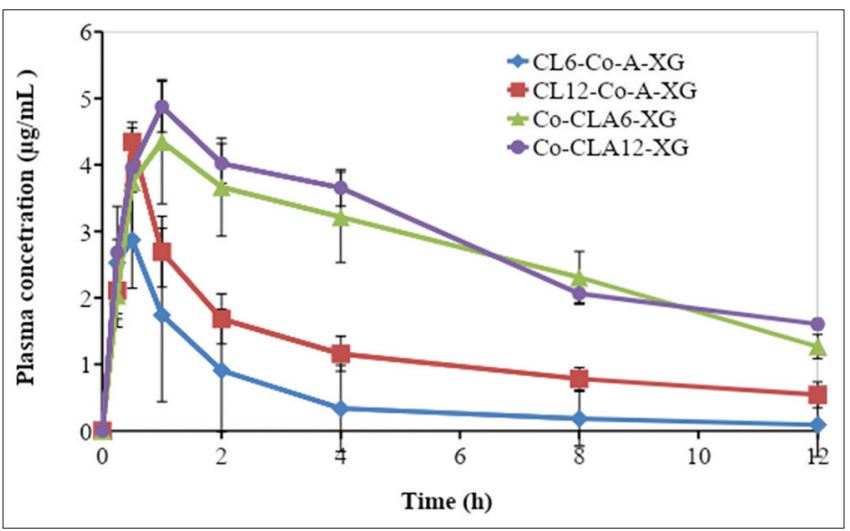

Fig. 2: Plasma concentration-time profiles after transdermal administration of diclofenac-containing hydrogel for Co-CLA6-XG (F2), Co-CLA12-XG (F4), CL6-Co-A-XG (F6), and CL12-Co-A-XG (F8), as the matrix. Green, purple, blue, and red lines represent F2, F4, F6, and F8, respectively. Each point represents the mean \pm standard error; $n=6$

diclofenac sodium as a drug model. The low partition coefficient of sodium diclofenac $(\log p=0.70)$ was an important factor related to drug permeation because the partition coefficient indicates the ability of a drug to partition into hydrophobic and hydrophilic phases [21].

\section{In vivo penetration study}

Fig. 2 shows the mean of the plasma concentration-time profiles of diclofenac after transdermal administration for $12 \mathrm{~h}$. The results show that the highest area under the curve (AUC) was achieved by F4 (CoCLA12-XG hydrogel), followed by the Co-CLA6-XG (F2), CL12-Co-A-XG (F8), and CL6-Co-A-XG (F6) matrices. However, the AUC value of F4 was not significantly different than that of $F 2$, ( $p>0.005)$. As presented 
in Table 5, the AUC, $\mathrm{C}_{m a x}, \mathrm{~T}_{\text {my, }}, \mathrm{t}_{1}$, and mean residence time (MRT) values of the transdermal diclofenac hydrogel, comprising Co-CLA6$\mathrm{XG}$ as a matrix (F2), showed no significant difference ( $p>0.005)$ with F4, comprising Co-CLA12-XG. In contrast, the AUC of the transdermal hydrogel F6 and F8, comprising CL6-Co-A-XG and CL12-Co-A-XG, respectively, significantly decreased. The results indicated that the crosslinking degree of amylose and the method of preparation the excipient affected their drug delivery. In addition, the MRT and $K_{\mathrm{e}}$ values of the transdermal hydrogels of F2, F4, and F8 indicated that the produced excipients can be used as a transdermal matrix with controlled and prolonged release behavior.

\section{CONCLUSION}

For diclofenac sodium as the model drug, the in vitro and in vivo penetration study results indicated the feasibility of developing hydrogels using Co-CLA6-XG, Co-CLA12-XG, CL6-Co-A-XG, or CL12-Co-A-XG as a matrix former that could be used as a good transdermal dosage form. The results also show the ability of these hydrogels to provide good controlled release permeation of the active model drug. Comparison of the in vivo and in vitro skin penetration data in this study was difficult, but the results of both studies suggested that the hydrogel formulations would provide good controlled drug permeation through the skin. Further studies are needed to determine if these hydrogels can be used for humans in clinical studies.

\section{CONFLICTS OF INTEREST}

The authors declare that they have no conflicts of interest.

\section{REFERENCES}

1. Ranade VV, Hollinger MA. Drug Delivery System. $2^{\text {nd }}$ ed. London: CRC Press; 2004.

2. Kadam AS, Ratnaparkhi MP, Chaudhary SP. Transdermal drug delivery : An overview. Int J Res Dev Pharm Life Sci 2014;3:1042-53.

3. Peppas NA, Buri PA. Surface, interfacial and molecular aspects of polymer bioadhesion on soft tissues. J Control Release 1985;2:257-75.

4. Nachaegari SK, Bansal AK. Coprocessed excipients for solid dosage forms. Pharm Tech 2004;28:52-64.

5. Onofre F, Wang YJ, Mauromoustakos A. Effects of structure and modification on sustained release properties of starches. Carbohydr Polym 2009;76:541-7.

6. Rowe RC, Sheskey PJ, Owen SC. Handbook of Pharmaceutical
Excipients. $5^{\text {th }}$ ed. Washington, DC: Pharmaceutical Press and American Pharmacist Association; 2006.

7. Surini S, Ariani L, Putri KS, Hayun H, Anwar E. Coprocessed excipients of crossliked amylose and xanthan gum for use in controlled release dosage forms. Int J App Pharm 2018;10:59-65.

8. Witt K, Bucks D. Studying in vitro skin penetration and drug release to optimize dermatological formulations. In: Pharmaceutical Technology. New York: Anvastar Communication Inc.; 2003.

9. Cury BS, Klein SI, Evangelista RC. Modeling a system of phosphated cross-linked high amylose for controlled drug release. Part 1: Synthesis and polymer characterization. React Funct Polym 2009;68:1200-6.

10. Marthur A. Studies on Phosphorylation Status of Starch in Potato Tubers (Solanum tuberosum L.). Dissertation. Patiala: Department of Biotechnology and Environmental Sciences Thapar Institute of Engineering and Technology Patiala; 2003.

11. Paschall FE. Phosphorylation with Inorganic Phosphate Salts. New York: Academic Press; 1964.

12. Klimes J, Sochor J, Dolezal P, Körner J. HPLC evaluation of diclofenac in transdermal therapeutic preparations. Int J Pharm 2001;217:153-60.

13. Sintov AC, Botner S. Transdermal drug delivery using microemulsion and aqueous systems: Influence of skin storage conditions on the in vitro permeability of diclofenac from aqueous vehicle systems. Int $\mathrm{J}$ Pharm 2006;311:55-62.

14. European Medicines Agency. Guideline on Bioanalytical Method Validation; 2011. Available from: http://www.ema.europa.eu/docs/en GB/document library/Scientific guideline/2011/08/WC500109686.pdf.

15. Murphy J, Riley JP. A modified single-solution method for the determination of phosphorus in natural waters. Anal. Chim. Acta 1962;27:31-6.

16. Bhanja S, Kishore P, Das AK, Secunderabad M, Pradesh A. Formulation and evaluation of diclofenac transdermal gel. J Adv Pharm Educ Res 2013;3:248-59.

17. Sherman P. Techniques for assessing the rheological properties of toiletry and cosmetic products. J Soc Cosmet Chem 1966;17:439-65.

18. Shargel L, Andrew BC, Wu-Pong S. Applied Biopharmaceutics and Pharmacokinetics. $5^{\text {th }}$ ed. United States: McGraw-Hill Access Pharmacy; 1993.

19. Dumoulin Y, Alex S, Szabo P, Cartilier L, Mateescu MA. Crosslinked amylose as matrix for drug controlled release. X-ray and FT-IR structural analysis. Carbohydr. Polym. 1998;37:361-70.

20. Lenaerts V, Beck RH, Van Bogaert E, Chouinard F, Höpcke R, Désévaux C. Cross-linked High Amylose Starch for Use in Controlledrelease Pharmaceutical Formulations and Processes for its Manufacture. United States Patent US No. 6, 607; 2003.

21. Fini A, Bassini G, Monastero A, Cavallari C. Diclofenak salts, VIII. Effect of the counterions on the permeation through porcine membrane from aqueous saturated solutions. Pharmaceutics 2012;4:413-29. 